\title{
An algorithm for preoperative differential diagnostics of parotid tumours on the basis of their dynamic and diffusion-weighted magnetic resonance images: a retrospective analysis of 158 cases
}

\author{
B. Mikaszewski ${ }^{1}$, K. Markiet ${ }^{2}$, A. Smugała ${ }^{3}$, D. Stodulski ${ }^{1}$, E. Szurowska², C. Stankiewicz ${ }^{1}$ \\ ${ }^{1}$ Department of Otolaryngology, Medical University of Gdansk, Poland \\ ${ }^{2} 2^{\text {nd }}$ Department of Radiology, Medical University of Gdansk, Poland \\ ${ }^{3}$ Department of Radiology, University Clinical Centre, Gdansk, Poland
}

[Received: 3 December 2017; Accepted: 19 December 2017]

Background: To verify the usefulness of a new algorithm for preoperative differential diagnostics of parotid tumours on the basis of their dynamic and diffusion-weighted magnetic resonance imaging (MRI).

Materials and methods: The retrospective analysis included 158 consecutive surgical patients with parotid tumours. Aside from ultrasound-guided fine needle biopsy, the protocol of preoperative evaluation included dynamic and diffusion-weighted MRI. According to the new diagnostic algorithm, the result of fine needle biopsy was considered only in the case of lesions with time to peak enhancement $\left(T_{\text {peak }}\right)>60 \mathrm{~s}$ and washout rate $(W R) \leq 30 \%$ on dynamic MRI and apparent diffusion coefficient $(A D C) \leq 1.7 \times 10^{3} \mathrm{~mm} / \mathrm{s}^{2}$ on diffusion-weighted $M R I$, or those presenting with concomitant lymphadenopathy. The accuracy of this algorithm was verified against final histopathological diagnoses.

Results: The new algorithm gave 10 true positive and 2 false positive results, as well as 132 and 14 true and false negative results, respectively. Its sensitivity and specificity (41.7\% and $98.5 \%$, respectively) were the same as in the case of fine needle biopsy alone. None of the 59 tumours that were qualified as benign solely on the basis of preoperative MRI turned out to be malignant on postoperative histopathological examination.

Conclusions: Interpreted together, dynamic and diffusion-weighted MRIs provide the same accuracy in preoperative differential diagnostics of parotid tumours as fine needle biopsy. This substantiates the use of diagnostic algorithms in which biopsy would serve mostly as a secondary test to verify selected ambiguous radiological diagnoses. (Folia Morphol 2018; 77, 1: 29-35)

Key words: pleomorphic adenoma, Warthin tumour, surgery, fine needle biopsy 


\section{INTRODUCTION}

Appropriate qualification for operation is a key component of surgical management. This issue becomes particularly important in the case of tumour resection. While most benign lesions can be subjected to local excision, malignant tumours require extended resection with a margin of normal tissue. Selection of an appropriate surgical technique and defining the extent of resection are also important in the case of parotid tumours, representing approximately $5 \%$ of all head and neck neoplasms [15]. The vast majority of lesions from this relatively heterogeneous group are pleomorphic adenomas and Warthin tumours, usually suitable for conservative or even expectant treatment. However, also primary or secondary malignancies may develop within the parotid gland; suboptimal resection of such lesions would have devastating consequences $[3,4]$.

Cytological evaluation of fine needle biopsy specimens constitutes a standard for preoperative differential diagnostics of parotid tumours [17]. However, a growing body of evidence suggest that this examination does not provide adequate diagnostic accuracy, especially in the case of small and/or deeply located lesions. In up to $5 \%$ of the cases, material obtained during biopsy of such tumours may be not representative enough to determine their character $[6,14,16,23]$. Moreover, an incorrectly performed biopsy may lead to various complications, including systemic spread of cancer cells and ascending infection of the head and neck $[2,9]$.

These potential drawbacks of fine needle biopsy stimulated interest in dynamic magnetic resonance imaging (MRI) and its potential application in the preoperative differential diagnostics of parotid tumours. The time intensity curves (TICs) for two most common histological types of benign parotid lesions, pleomorphic adenomas and Warthin tumours, differ from those for parotid malignancies $[1,7-13,19]$. A few studies applying this knowledge in differential diagnostics of parotid tumours produced promising results $[18,20,21]$. However, this evidence, originating from relatively small studies of patients with pleomorphic adenomas, Warthin tumours and parotid malignancies, was not confirmed in routine clinical practice [5]. Moreover, some empirical data imply that parotid malignancies are not homogeneous in terms of their presentation on dynamic MRI, and therefore some of them may be misdiagnosed as pleomorphic adenomas [20].
Since 2013, all patients with parotid tumours treated surgically at our centre have been subjected to preoperative dynamic and diffusion-weighted MRI aside from routine fine needle biopsy. Therefore, we have collected a large database of patients in whom the result preoperative MRI can be compared with final histopathological diagnosis. Similar to previous studies, our earlier analyses of this material were limited to comparative analysis of MRI parameters for pleomorphic adenomas, Warthin tumours and parotid malignancies. We showed that dynamic MRI is highly accurate in differential diagnostics of Warthin tumours and parotid malignancies, but it is diffusionweighted MRI which provides optimum accuracy in differentiating pleomorphic adenomas from malignant lesions. On the basis of these data, we have developed an algorithm for preoperative differential diagnostics of parotid tumours based on their presentation on dynamic and diffusion-weighted MRI; in this algorithm, the role of fine needle biopsy was limited to an adjunct examination performed solely in ambiguous cases (Fig. 1). The aim of this study was to verify the usefulness of this algorithm in a retrospective analysis of more than 150 patients with parotid tumours. Contrary to previous studies, we analysed all consecutive cases treated at our centre, also benign lesions with histological types other than pleomorphic adenoma or Warthin tumour.

\section{MATERIALS AND METHODS}

The retrospective analysis included 158 consecutive patients with parotid tumours treated surgically at the Department of Otolaryngology, Medical University of Gdansk, between May 2013 and February 2015. Aside from ultrasound-guided fine needle biopsy, the protocol of preoperative evaluation included also dynamic and diffusion-weighted MRI. However, the principal criterion used to determine the type and extent of surgical resection was a result of fine needle biopsy. Final diagnosis was established on the basis of histopathological examination of surgical specimen. During the retrospective analysis, however, we compared the accuracy of true preoperative diagnoses with a hypothetic accuracy of the diagnoses that could be established with an aid of the algorithm described below, based on the results of dynamic and diffusion-weighted MRI.

The protocol of the study was approved by the Local Bioethics Committee at the Medical University of Gdansk, and all participants gave their written 


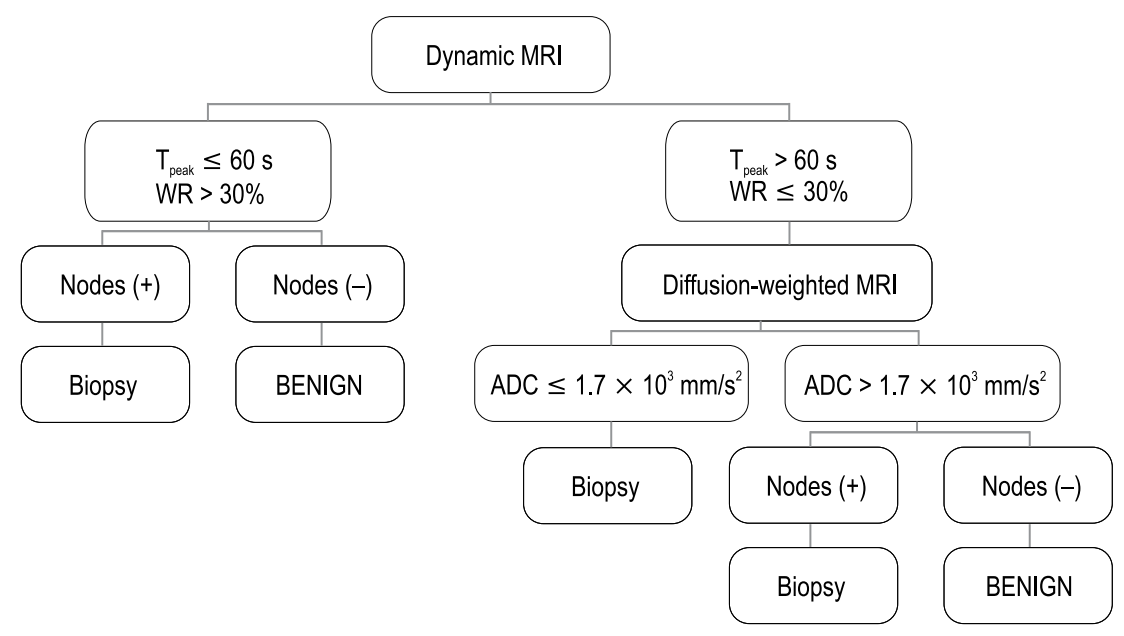

Figure 1. A scheme illustrating the role of dynamic and diffusion weighted magnetic resonance imaging (MRI) and fine needle biopsy in preoperative differential diagnostics of parotid tumours; $A D C$ — apparent diffusion coefficient; $W R$ — washout rate; $T_{\text {peak }}$ - time to peak enhancement.

informed consent for all medical procedures, as well as for the use of their data for research purposes.

\section{Dynamic and diffusion-weighted MRI}

Magnetic resonance examinations were performed in a 1.5-T scanner (Magnetom Area, Siemens, Erlangen, Germany) with the use of a head coil. Pre- and post-contrast T1-weighted spin-echo (repetition time ms/echo time ms, 659/12; $256 \times 256$ matrix; 5-mm section thickness; $2.5-\mathrm{mm}$ intersection gap; $20-25-\mathrm{cm}$ field of view; one signal acquired) and pre-contrast T2-weighted fast spin-echo (repetition time ms/echo time ms, 3680/93; $256 \times 256$ matrix; 5-mm section thickness; 2.5-mm intersection gap; $20-25-\mathrm{cm}$ field of view; one signal acquired) images were obtained in three orthogonal planes. Subsequently, diffusion weighted imaging was performed with a multisection spin-echo single-shot echoplanar sequence in the transverse plane $(3200 / 118,20-25-\mathrm{cm}$ field of view, $128 \times 128$ matrix, 5-mm section thickness, 2.5-mm intersection gap, two signals acquired, 48-s acquisition time). Sensitising diffusion gradients were applied sequentially in the $x, y$, and $z$ directions with $b$ values of 0,500 , and $1000 \mathrm{~s} / \mathrm{mm}^{2}$. Apparent diffusion coefficient $(A D C)$ maps were generated. During examinations tumours were identified on the transverse T1-weighted images, and three sections, including one section that accounted for the maximum diameter of the tumour, were selected for dynamic contrast-enhanced MRI (300/13, 20-25-cm field of view, $256 \times 256$ matrix, 5-mm section thickness, 2.5-mm intersection gap, one signal acquired). Gadobutrol (Gadovist, Bayer Schering Pharma, Berlin, Germany) was administered intravenously at a rate of $2-3 \mathrm{~mL} / \mathrm{s}$ (total dose, $0.1 \mathrm{mmol} / \mathrm{kg}$ of body weight), followed by a 20-mL saline flush. MRIs were sequentially obtained before and $0,30,60,90,120,150,180,210,240$, 270 , and $300 \mathrm{~s}$ after contrast agent administration. First observer manually identified regions of interest (ROIs) for signal intensity measurements to avoid vessels and cystic parts of the tumours and constructed TICs with the use of dedicated software. All MRIs were interpreted in consensus by two observers who were blinded to clinical information and biopsy results. For ADC measurements, the same observer manually placed ROls within a given lesion. Each time, three ROls were placed within the area that corresponded to one used for TICs construction, and the obtained $A D C$ values were averaged. ADC was calculated with linear regression analysis.

\section{Cytological and histological examination}

Both the material obtained during preoperative fine needle biopsy and the surgical specimens of parotid tumours were processed routinely, stained with haematoxylin and eosin and subjected to cytological and histological examination under a light microscope.

\section{Algorithm for differential diagnostics}

During the first stage, we identified benign lesions presenting with Warthin tumour-like pheno- 
type on dynamic MRI. The lesions with time to peak enhancement $\left(T_{\text {peak }}\right) \leq 60 \mathrm{~s}$ and washout rate (WR) $>30 \%$ were considered benign, except for those in the case of which the presence of concomitant lymphadenopathy raised a suspicion of malignant transformation; in such cases, final preoperative diagnosis was established with fine needle biopsy. During the second stage, on the basis of ADC > $1.7 \times 10^{3}$ $\mathrm{mm} / \mathrm{s}^{2}$ documented on diffusion-weighted MRI, we identified the masses presenting with pleomorphic adenoma-like phenotype. Similar to the first stage, a result of fine needle biopsy was considered the final preoperative diagnosis in the case of lesions with $A D C$ $>1.7 \times 10^{3} \mathrm{~mm} / \mathrm{s}^{2}$ and concomitant lymphadenopathy. Consequently, fine needle biopsy was used to establish the final preoperative diagnosis for tumours with $\mathrm{T}_{\text {peak }}>60 \mathrm{~s}, \mathrm{WR} \leq 30 \%$ and $\mathrm{ADC} \leq 1.7 \times 10^{3}$ $\mathrm{mm} / \mathrm{s}^{2}$, as well as for the remaining lesions presenting with concomitant lymphadenopathy (Fig. 1).

\section{Statistical analysis}

During statistical analysis, the accuracy of truly used diagnostic algorithm based on fine needle biopsy was compared with the accuracy of the algorithm presented above, based on the results of dynamic and diffusion-weighted MRI. Sensitivity and specificity of both algorithms in identification of parotid malignancies were calculated, along with the areas under their ROC curves (AUCs, with their 95\% confidence intervals $[95 \% \mathrm{Cl}]$ ). True/false positive and negative rates were calculated in relation to a gold standard, the result of final histopathological examination. All calculations were carried out with Statistica 10 package (StatSoft, Tulsa OK, USA), with the threshold of statistical significance set at $p \leq 0.05$.

\section{RESULTS}

Histopathological examination of the surgical specimens showed that the analysed material included 24 parotid malignancies (15\%) and 134 benign or non-neoplastic lesions (85\%). The group of malignancies included adenocarcinomas $(n=6)$, myoepithelial carcinomas $(n=4)$, metastases from other sites $(n=4)$, mucoepidermoid carcinomas $(n=2)$, adenoid cystic carcinomas $(n=2)$, acinic cell carcinomas $(n=2)$, lymphomas $(n=2)$, ductal carcinoma $(n=1)$ and planoepithelial carcinoma $(n=1)$. The group of benign tumours and non-neoplastic lesions was comprised of pleomorphic adenomas $(n=57)$, Warthin tumours $(n=52)$ and sporadic cases of other tumours, cysts, inflammatory lesions, etc. $(n=25)$.

Based on the result of fine needle biopsy, 12 (8\%) parotid lesions were identified as malignant tumours. This group included 10 true positive diagnoses and 2 false positive ones, which corresponded to $41.7 \%$ sensitivity. The number of true and false negative diagnoses was 132 and 14 , respectively, corresponding to $98.5 \%$ specificity. The area under ROC curve for fine needle biopsy was $0.701(95 \% \mathrm{Cl} 0.566-0.836)$.

A total of $28(18 \%)$ lesions were considered benign on the basis of preoperative dynamic MRI ( $T_{\text {peak }} \leq 60 \mathrm{~s}$ and WR $>30 \%$ ). Due to presence of concomitant lymphadenopathy, final preoperative diagnosis for 13 out of the $28(46 \%)$ potentially benign masses was established on the basis of fine needle biopsy. In all these cases, cytological diagnosis was consistent with the radiological one, confirming benign character of the lesions. However, one tumour qualified as benign on the basis of both dynamic MRI and fine needle biopsy eventually turned out to be malignant on postoperative histopathological examination ( 1 false negative result). When the results of diffusion-weighted MRI (ADC > $1.7 \times 10^{3}$ $\mathrm{mm} / \mathrm{s}^{2}$ ) were applied, 44 out of the 130 lesions non-classified on the basis of dynamic MRI (34\%) were identified as benign. This subset included $13(30 \%)$ lesions with concomitant lymphadenopathy, in the case of which the final preoperative diagnosis was established on the basis of fine needle biopsy. In one of these cases, a malignancy was identified during examination of the biopsy specimen. In the remaining 12 cases, the result of cytological examination was consistent with the diagnosis established on diffusion-weighted MRI. Preoperative diagnoses established on the basis of diffusion-weighted MRI and fine needle biopsy were in complete agreement with the results of postoperative histopathological examination. Fine needle biopsy was used to establish the final preoperative diagnoses for the remaining 86 lesions. In 11 (13\%) cases, a malignancy was found on cytological examination. In 9 out of the 11 tumours, this diagnosis was consistent with the result of final histopathological examination ( 2 false positive results). However, the subset of 75 lesions that were diagnosed as benign during preoperative cytological examination included as many as 13 tumours that eventually turned out to be malignant (13 false negative results). All these data are presented schematically on Figure 2 . 


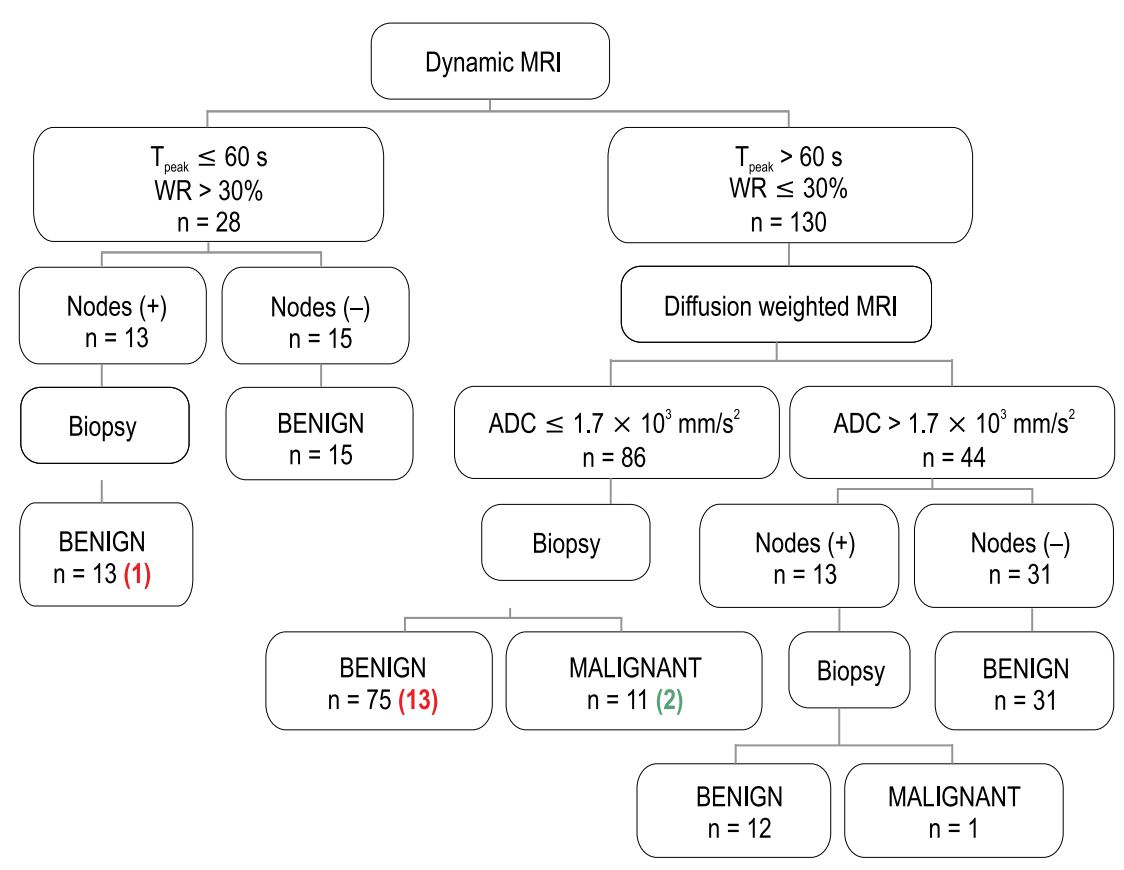

Figure 2. Results of preoperative differential diagnostics with the algorithm based on the results of dynamic and diffusion-weighted magnetic resonance imaging (MRI). Numbers in parentheses represent false negative (highlighted in red) and false positive diagnoses (highlighted in green); $A D C$ - apparent diffusion coefficient; $W R$ - washout rate; $T_{\text {peak }}$ - time to peak enhancement.

Overall, the algorithm based on the results of dynamic and diffusion-weighted MRI supported with fine needle biopsy gave 10 true positive and 2 false positive results, as well as 132 and 14 true and false negative results, respectively. This corresponded to $41.7 \%$ sensitivity and $98.5 \%$ specificity of the algorithm in differential diagnostics of parotid tumours. The area under ROC curve for the algorithm was 0.701 (95\% Cl 0.566-0.836). All measures of diagnostic accuracy mentioned above were exactly the same as those calculated for fine needle biopsy alone.

The subset of 14 parotid malignancies, in the case of which the hereby presented diagnostic algorithm produced false negative results, included 11 lesions with concomitant lymphadenopathy. Lymphadenopathy was in turn absent in the case of two benign lesions for which our algorithm produced false positive results.

\section{DISCUSSION}

Potential application of dynamic MRI to the differential diagnostics of parotid tumours was postulated already more than two decades ago. A number of authors showed that the shape of TIC can be used to distinguish parotid malignancies, characterised by an early enhancement and a gradual washout of the contrast (i.e. short $T_{\text {peak }}$ and high WR) from pleomorphic adenomas (with a gradual enhancement and a slow washout, i.e. long $T_{\text {peak }}$ and high WR) and Warthin tumours (with an early enhancement and a rapid washout, i.e. short $\mathrm{T}_{\text {peak }}$ and low WR) $[1,7-10,12,13,19]$. However, further research on the cut-off values for $T_{\text {peak }}$ and WR providing maximum accuracy in the differential diagnostics of these three groups produced conflicting results. While there is a consensus with regards to the cut-off values for $T_{\text {peak }}$ and WR that accurately distinguish parotid malignancies from Warthin tumours $\left(T_{\text {peak }}=60 \mathrm{~s}\right.$ and $W R=30 \%)[9,18,20,21]$, the differential diagnostics of pleomorphic adenomas and malignant lesions is still a challenge. Clinical experience suggests that the previously proposed cut-off values for $T_{\text {peak }}(120,210$ or $240 \mathrm{~s})$ and WR (10\%) $[9,18,20,21]$ are not accurate enough to distinguish malignant tumours from pleomorphic adenomas. Moreover, the TICs for some parotid malignancies resemble those traditionally assigned to pleomorphic adenomas [20]. Therefore, some authors postulated that these two types of lesions should be distinguished on the basis of their ADC values determined on diffusion-weighted MRI. Previous studies identified two cut-off values for ADC providing 
optimum accuracy in the differential diagnostics of pleomorphic adenomas and parotid malignancies: $1.4 \times 10^{3} \mathrm{~mm}^{2} / \mathrm{s}$ and $1.8 \times 10^{3} \mathrm{~mm}^{2} / \mathrm{s}[5,22]$.

\section{Limitations of the study}

An important limitation of all the studies mentioned above was small number of examined parotid tumours. Having access to markedly larger group of patients, we were able to verify diagnostic accuracy of the previously proposed cut-off values for MRI parameters, and to develop our own diagnostic algorithm, presented in this paper. Moreover, we observed that most parotid malignancies were associated with concomitant lymphadenopathy, and inclusion of this parameter in our diagnostic algorithm resulted in further increase in its accuracy. These yet unpublished observations were confirmed in this study, as the accuracy of our algorithm turned out to be exactly the same as the accuracy of fine needle biopsy, still considered a gold standard for preoperative diagnostics of parotid tumours. Although we still used fine needle biopsy to verify some of the radiological diagnoses, the subset of 72 parotid tumours that were identified as benign solely on the basis of dynamic ( $n=28)$ or diffusion-weighted MRI $(n=44)$ included only two masses that eventually turned out to be malignant. The remaining false negative diagnoses $(n=13)$ were established in the case of masses that were considered potentially malignant on the basis of preoperative MRI but were eventually qualified as benign based on the result of fine needle biopsy.

Consequently, a question arises whether dynamic and diffusion-weighted MRI may replace fine needle biopsy in the preoperative differential diagnostics of parotid tumours? The list of arguments for routine use of dynamic and diffusion-weighted MRI includes more objective character of diagnostic imaging, less steep learning curve and lower risk of complications than in the case of fine needle biopsy. However, it should be remembered that imaging studies are with no doubt markedly more expensive than the biopsy and their availability is typically limited to tertiary centres. Moreover, also dynamic and diffusion-weighted MRI is associated with some subjectivity, for example, associated with the selection of ROI. Furthermore, one should remember that it was fine needle biopsy which enabled us to verify a false negative diagnosis established on the basis of diffusion-weighted MRI in one of the hereby presented cases. Altogether, this suggests that with the current state of knowledge, dynamic and diffusion-weighted MRI and fine nee- dle biopsy should be considered as complementary examinations in differential diagnostics of parotid tumours. Nevertheless, the results of this study imply also that due to its comparative accuracy but potentially greater invasiveness, fine needle biopsy could be used predominantly as a secondary test to verify selected ambiguous radiological diagnoses.

Our findings point also to directions of future research on the optimisation of differential diagnostics of parotid tumours. Such studies should centre around further improvement of algorithms based on dynamic and diffusion-weighted MRI, e.g. by inclusion additional clinical parameters. Such direction of future research seems justified since most false negative and false positive diagnoses established in our study could be corrected if the presence of concomitant lymphadenopathy or lack thereof were considered. Our experience suggests that also other clinical and demographic parameters, e.g. involvement of the deep parotid lobe and older age of the patient, may imply malignant character of a tumour. Another domain of future research should be comparative cost-effectiveness analysis of fine needle biopsy, dynamic and diffusion-weighted MRI and diagnostic algorithms combining all these methods. In other words, one should verify if greater accuracy of preoperative diagnostics, achieved due to implementation of novel MRI technologies, is reflected by a reduction of treatment costs.

\section{CONCLUSIONS}

Interpreted together, the results of dynamic and diffusion-weighted MRI provide the same accuracy in preoperative differential diagnostics of parotid tumours as fine needle biopsy. Taking into account previously documented drawbacks of the latter, this observation substantiates the use of diagnostic algorithms in which biopsy would serve mostly as a secondary test to verify selected ambiguous radiological diagnoses.

\section{REFERENCES}

1. Asaumi Jl, Shigehara H, Konouchi H, et al. Assessment of carcinoma in the sublingual region based on magnetic resonance imaging. Oncol Rep. 2002; 9(6): 1283-1287, indexed in Pubmed: 12375035.

2. Batsakis JG, el-Naggar AK. Warthin's tumor. Ann Otol Rhinol Laryngol. 1990; 99(7 Pt 1): 588-591, doi: 10.117 7/000348949009900719, indexed in Pubmed: 2195965.

3. Donovan DT, Conley JJ. Capsular significance in parotid tumor surgery: reality and myths of lateral lobectomy. Laryngoscope. 1984; 94(3): 324-329, doi: 10.1288/00005537198403000-00006, indexed in Pubmed: 6321863. 
4. Dykun RJ, Deitel M, Borowy ZJ, et al. Treatment of parotid neoplasms. Can J Surg. 1980; 23(1): 14-19, indexed in Pubmed: 6244883.

5. Eida S, Sumi M, Sakihama N, et al. Apparent diffusion coefficient mapping of salivary gland tumors: prediction of the benignancy and malignancy. AJNR Am J Neuroradiol. 2007; 28(1): 116-121, indexed in Pubmed: 17213436.

6. Flezar M, Pogacnik A. Warthin's tumour: unusual vs. common morphological findings in fine needle aspiration biopsies. Cytopathology. 2002; 13(4): 232-241, indexed in Pubmed: 12269895.

7. Hisatomi M, Asaumi Ji, Konouchi $\mathrm{H}$, et al. Assessment of dynamic MRI of Warthin's tumors arising as multiple lesions in the parotid glands. Oral Oncol. 2002; 38(4): 369-372, doi: 10.1016/S1368-8375(01)00073-2, indexed in Pubmed: 12076701.

8. Hisatomi M, Asaumi Ji, Yanagi $Y$, et al. Assessment of pleomorphic adenomas using MRI and dynamic contrast enhanced MRI. Oral Oncol. 2003; 39(6): 574-579, doi: 10.1016/ S1368-8375(03)00040-X, indexed in Pubmed: 12798400.

9. Matsuzaki $H$, Yanagi $Y$, Hara $M$, et al. Diagnostic value of dynamic contrast-enhanced MRI in the salivary gland tumors. Oral Oncol. 2007; 43(9): 940-947, doi: 10.1016/j.oraloncology.2006.11.009, indexed in Pubmed: 17257881

10. Ikarashi $F$, Nakano $Y$, Nonomura $N$, et al. Radiological findings of adenolymphoma (Warthin's tumor). Auris Nasus Larynx. 1997; 24(4): 405-409, doi: 10.1016/S03858146(97)10007-4, indexed in Pubmed: 9352834.

11. Ikeda M, Motoori K, Hanazawa T, et al. Warthin tumor of the parotid gland: diagnostic value of MR imaging with histopathologic correlation. AJNR Am J Neuroradiol. 2004; 25(7): 1256-1262, indexed in Pubmed: 15313720.

12. Joe VQ, Westesson PL. Tumors of the parotid gland: MR imaging characteristics of various histologic types. AJR Am J Roentgenol. 1994; 163(2): 433-438, doi: 10.2214/ ajr.163.2.8037045, indexed in Pubmed: 8037045.

13. Park J, Inoue S, Ishizuka $Y$, et al. [Salivary gland masses: dynamic MR imaging and pathologic correlation]. Nihon Igaku Hoshasen Gakkai Zasshi. 1997; 57(9): 581-585, indexed in Pubmed: 9293756.

14. Parwani AV, Ali SZ. Diagnostic accuracy and pitfalls in fine-needle aspiration interpretation of Warthin tumor.
Cancer. 2003; 99(3): 166-171, doi: 10.1002/cncr.11207, indexed in Pubmed: 12811857.

15. Przewoźny T, Stankiewicz C. Neoplasms of the parotid gland in northern Poland, 1991-2000: an epidemiologic study. Eur Arch Otorhinolaryngol. 2004; 261 (7): 369-375, doi: 10.1007/s00405-003-0698-4, indexed in Pubmed: 14586626.

16. Que Hee CG, Perry CF. Fine-needle aspiration cytology of parotid tumours: is it useful? ANZ J Surg. 2001; 71(6): 345-348, doi: 10.1046/j.1440-1622.2001.02121.x, indexed in Pubmed: 11409019.

17. Stewart CJ, MacKenzie K, McGarry GW, et al. Fine-needle aspiration cytology of salivary gland: a review of 341 cases. Diagn Cytopathol. 2000; 22(3): 139-146, indexed in Pubmed: 10679992.

18. Takashima S, Noguchi Y, Okumura T, et al. Dynamic MR imaging in the head and neck. Radiology. 1993; 189(3): 813-821, doi: 10.1148/radiology.189.3.8234709, indexed in Pubmed: 8234709.

19. Traxler $M$, Hajek $P$, Solar $P$, et al. Magnetic resonance in lesions of the parotid gland. Int J Oral Maxillofac Surg. 1991; 20(3): 170-174, indexed in Pubmed: 1890326.

20. Tsushima $Y$, Matsumoto $M$, Endo $K$, et al. Characteristic bright signal of parotid pleomorphic adenomas on T2-weighted MR images with pathological correlation. Clin Radiol. 1994; 49(7): 485-489, indexed in Pubmed: 8088045.

21. Yabuuchi H, Fukuya T, Tajima T, et al. Salivary gland tumors: diagnostic value of gadolinium-enhanced dynamic MR imaging with histopathologic correlation. Radiology. 2003; 226(2): 345-354, doi: 10.1148/radiol.2262011486, indexed in Pubmed: 12563124.

22. Yabuuchi $H$, Matsuo $Y$, Kamitani $T$, et al. Parotid gland tumors: can addition of diffusion-weighted MR imaging to dynamic contrast-enhanced MR imaging improve diagnostic accuracy in characterization? Radiology. 2008; 249(3): 909-916, doi: 10.1148/radiol.2493072045, indexed in Pubmed: 18941162.

23. Zbären $P$, Schär $C$, Hotz MA, et al. Value of fine-needle aspiration cytology of parotid gland masses. Laryngoscope. 2001; 111(11 Pt 1): 1989-1992, doi: 10.1097/00005537200111000-00023, indexed in Pubmed: 11801984. 